\title{
Epigenetic consequences of artificial reproductive technologies to the bovine imprinted genes SNRPN, H19/IGF2, and IGF2R
}

\author{
Lawrence C. Smith ${ }^{1}$, Jacinthe Therrien ${ }^{1}$, France Filion ${ }^{1}$, Fabiana Bressan ${ }^{2}$ and Flávio V. Meirelles ${ }^{2}$ \\ Department of Veterinary Biomedicine, Centre de Recherche en Reproduction Animale, Faculty of Veterinary Medicine, University of Montreal, \\ Saint-Hyacinthe, QC, Canada \\ ${ }^{2}$ Department of Veterinary Medicine, Faculty of Animal Sciences and Food Engineering, University of São Paulo, Pirassununga, Brazil
}

Edited by:

Alex Bach, Institut de Recerca i Tecnologia Agroalimentaries, Spain

Reviewed by:

David MacHugh, University College Dublin, Ireland

Jiuzhou Song, University of

Maryland, USA

*Correspondence:

Lawrence C. Smith, Department of Veterinary Biomedicine, Centre de Recherche en Reproduction

Animale, Faculty of Veterinary Medicine, University of Montreal, 3200 Sicotte, Saint-Hyacinthe, QC J2S 7C6, Canada

e-mail:smithl@medvet.

umontreal.ca
Animal breeders have made widespread use of assisted reproductive technologies to accelerate genetic improvement programs aimed at obtaining more, better and cheaper food products. Selection approaches have traditionally focused on Mendel's laws of inheritance using parental phenotypic characteristics and quantitative genetics approaches to choose the best parents for the next generation, regardless of their gender. However, apart from contributing DNA sequence variants, male and female gametes carry parentalspecific epigenetic marks that play key roles during pre- and post-natal development and growth of the offspring. We herein review the epigenetic anomalies that are associated with artificial reproductive technologies in current use in animal breeding programs. For instance, we demonstrate that bovine embryos and fetuses derived by in vitro culture and somatic cell nuclear transfer show epigenetic anomalies in the differentially methylated regions controlling the expression of some imprinted genes. Although these genomic imprinting errors are undetected in the somatic tissues after birth, further research is warranted to examine potential germ cell transmission of epimutations and the potential risks of reproducing cattle using artificial reproductive technologies.

Keywords: epigenetics, genomic imprinting, in vitro culture, nuclear transfer, animal cloning, cattle

\section{INTRODUCTION}

Artificial reproductive technologies (ART) have been widely applied to improve the fertility of domestic species, particularly in bovine livestock production systems. From the less invasive ART of artificial insemination using frozen semen to the most recent advances in animal cloning by somatic cell nuclear transfer (SCNT), few studies have focused on the potential long term consequences, both positive and negative, of reproductive technologies to domestic animal health and welfare (Urrego et al., 2014). Moreover, scientific evidence for transgenerational inheritance of features acquired from the environment during lifetime, e.g., food intake, is mounting and it is possible that such epigenetic phenomena could affect animal production and reproduction traits for several generations. Examples of epigenetic inheritance induced by genetic (Morgan et al., 1999; Grandjean et al., 2009) and environmental ( $\mathrm{Ng}$ et al., 2010) perturbations have been shown in rodents, and the mechanisms involved have begun to be elucidated (Daxinger and Whitelaw, 2012; Heard and Martienssen, 2014). Furthermore, in humans, retrospective cohort studies demonstrate transgenerational effects of prenatal famine exposure on birth weight and metabolic disease rates (Pembrey et al., 2006; Painter et al., 2008; Lumey et al., 2009), suggesting that epigenetic modifications caused by metabolic changes to domestic animals may also be transgenerationally inherited.
The role of epigenetics and, particularly of imprinted genes, in domestic animal production traits remains largely unknown. According to recent information obtained on the Geneimprint website (www.geneimprint.org), which provides a listing of genes by species, only a few imprinted genes have been identified in domestic species (cow, $n=19$; sheep, $n=17$; pig, $n=22$ ) compared to the much larger number already identified in humans $(n=95)$ and mice $(n=123)$. Nonetheless, imprinted genes have been found in livestock to affect traits such as milk yield, growth and carcass quality, fat and meat deposition and fetal development. For instance, the paternally expressed imprinted gene insulin-like growth factor 2 (IGF2) has been associated with meat traits and body weight in beef cattle (Flisikowski et al., 2007; Goodall and Schmutz, 2007; Sherman et al., 2008; Bagnicka et al., 2010), and muscle mass and fat deposition in pigs (Van Laere et al., 2003; Vykoukalova et al., 2006; Stinckens et al., 2010). Considering the important role IGF2 and other imprinted genes have on a number of production traits, it is likely that the inclusion of genomic imprinting in the calculation of breeding values using traditional phenotypic records (pedigreebased selection) and the more recent use of DNA polymorphisms (genomic selection) will be useful to improve animal selection schemes, as indicated by timely recent review articles on this topic (Goddard and Whitelaw, 2014; Magee et al., 2014). 
Animal cloning by SCNT has highlighted the importance of epigenetics on the phenotype. Although clones carry genetically identical chromosomes, wide-ranging phenotypic variations have been identified in a number of growth parameters, particularly during prenatal stages of life, when epigenetic errors can lead to the aberrant expression of genes that play a key role in the development and differentiation of both embryonic and extraembryonic tissues. Nonetheless, high variability of body size has been observed in bovine clones derived by nuclear transfer, suggesting that epigenetic errors may also play a role after birth (Gärtner et al., 1998; Heyman et al., 2004). Since cloned animals carry epimutations to a number of imprinted genes, it is possible that such epigenetic marks could be transmitted through their germ cells to the following generations.

\section{DEVELOPMENTAL ANOMALIES OBSERVED IN ANIMAL CLONING ARE SIMILAR TO THOSE OBSERVED IN GESTATIONS FROM IN VITRO PRODUCED EMBRYOS}

The low survival and high morbidity of cloned offspring has become a major obstacle for a wide scale commercial implementation of the SCNT methodology (Smith et al., 2012). The Food and Drug Agency of the USA government has compiled thousands of data from cloned cows, pigs and goat to conclude that the food products from cloned animals and from their offspring are not different from naturally bred animals and, therefore, carry no health risk for human consumption. Nonetheless, public perception has rendered consumers reticent and few retailers are willing to introduce cloned animal products in the food chain. Phenotypic abnormalities associated with somatic cell cloning have been described in detail by numerous laboratories worldwide (Heyman et al., 2002; Wells et al., 2004), including recent reports describing the various neonatal clinical outcomes observed in cloned progeny of both Bos taurus (Brisville et al., 2011; Buczinski et al., 2011; Kohan-Ghadr et al., 2011) and Bos indicus cattle (Meirelles et al., 2010).

\section{EPIGENETIC REPROGRAMMING OF GENOMIC IMPRINTS DURING GAMETOGENESIS AND EARLY EMBRYONIC DEVELOPMENT}

Epigenetics is the hereditary variation in genomic activity that is independent of any alteration of the DNA sequence. Epigenetic inheritance refers to the memory of such activity; transferred between cellular generations through mitosis, and between organismal generations through meiosis and, therefore, is a link between genotype and phenotype that controls the expression of a locus. Several different types of epigenetic modifications contribute to stabilize gene expression in specialized cell types so that cellular identity and lineage fidelity is preserved, including those that alter chromatin structure, modify DNA and histones, remodel nucleosomes and incorporate variant histones. However, epigenetic stability is reprogrammed globally in two phases of the life cycle: in primordial germ cells (PGC) during gametogenesis and then in the zygote immediately after fertilization. The widespread epigenetic reprogramming in PGCs is associated with a global demethylation and is thought to be important for preventing the transmission of inappropriate epigenetic information to the next generation. Although zygotic demethylation seems to occur globally, some genomic sequences, including imprinted genes, maintain methylation during preimplantation and are essential for proper development. Moreover, some histones are retained at non-random genomic locations in the sperm (Wykes and Krawetz, 2003) and can be inherited in the zygote (van der Heijden et al., 2008), thus allowing that the protection of DNA methylation at some imprints enables the inheritance of histone modifications from the gametes to the next generation.

Genomic imprinting is defined as a process by which certain genes are expressed differentially according to the parent-oforigin and is, therefore, independent of the classical Mendelian inheritance model. Both the establishment and the maintenance of genomic imprints during embryogenesis are essential for the proper embryonic and placental development. For instance, the imprinted gene H19 (non-protein coding) is silenced on the paternal allele, thus enabling IGF2 paternal expression. On the other hand, $\mathrm{H} 19$ is expressed from the non-silenced maternal allele where it blocks IGF2 maternal expression. In contrast, the small nuclear ribonucleaoprotein polypeptide (SNRPN) gene is silenced on the maternal allele and, thus, is monoallelically expressed from the paternal allele. Moreover, the receptor for IGF2 (IGF2R) is transcribed exclusively from the maternal allele due to the repression of paternal transcription by the non-coding AIR transcript. The epigenetic processes of DNA methylation and histone modulation are both involved in achieving monoallelic expression of imprinted genes. The numerous human genetic diseases associated with imprinted genes, including Beckwith-Wiedemann syndrome (BWS; Brown et al., 1996), Silver-Russel syndrome (Bartholdi et al., 2009), Angelman syndrome (Runte et al., 2004) and Prader-Willi syndrome (Reed and Leff, 1994), evidence the importance of allele-specific expression patterns of imprinted genes. Interestingly, risks for some of the above syndromes are elevated in children conceived by ART (Maher et al., 2003), suggesting that the exposure of gametes and/or early embryos to in vitro environments may cause epigenetic alterations to imprinted genes.

\section{EPIGENETIC REPROGRAMMING OF GENOMIC IMPRINTS IN CLONED EMBRYOS AND FETUSES}

A common condition in ruminants derived from ARTs is the large offspring syndrome, or LOS (Young et al., 1998; Ceelen and Vermeiden, 2001), which correlates with IGF2R imprinting disruption (Young et al., 2001) and is considered as reminiscent of and therefore a suitable model for the BWS in humans (Chen et al., 2013). As described above (Developmental anomalies observed in animal cloning are similar to those observed in gestations from in vitro produced embryos), LOS commonly presents placental perturbations, leading to high birth weights and reduced survival rates. The incidence of such placental failures is especially important in cloning by nuclear transfer and IVF, representing the major cause of pregnancy failure in these animals (Heyman et al., 2002; Hill, 2002; Constant et al., 2006).

We have identified the DMRs of a series of imprinted genes that could potentially be implicated with placental development and the LOS phenotype in cattle. Using a bovine F1 hybrid model 
Day 17


Day 40
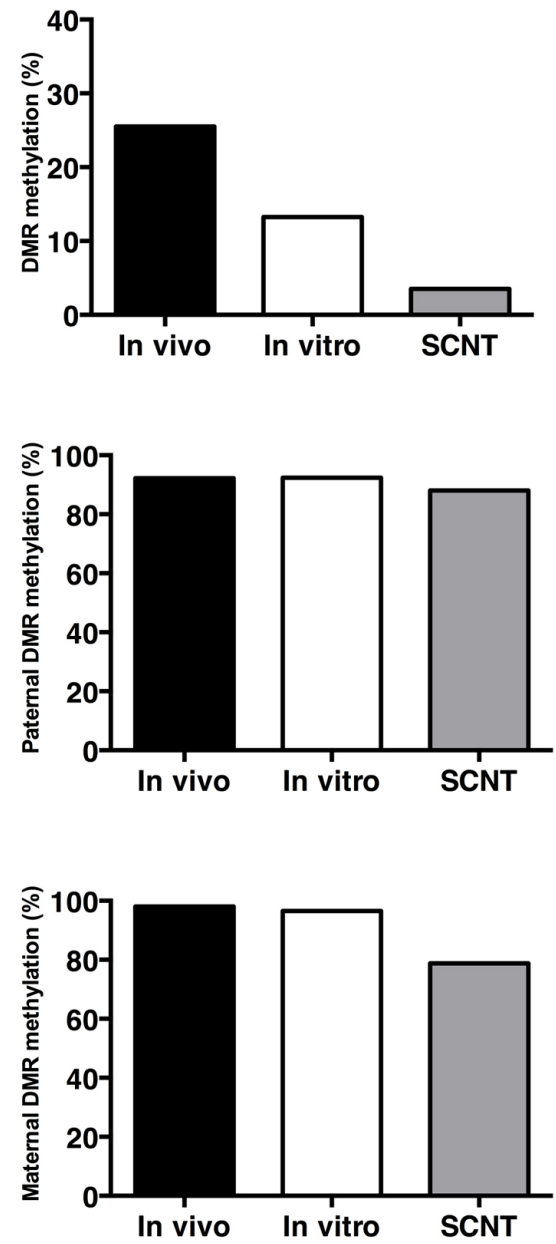

FIGURE 1 | DNA methylation levels of the DMRs from bovine imprinted genes SNRPN, H19/IGF2, and IGF2R in embryos (day 17) and early foetuses (day 40) derived in vivo (back bar), in vitro (white bar) and by SCNT (grey bar).

(Bos taurus vs. Bos indicus), we were able to identify SNPs in proximity to the DMR and exonic polymorphisms that enabled analysis of the imprinting status of several genes, including the maternally expressed H19 and IGF2R genes, and the paternally expressed SNRPN gene. Analysis was performed on day-17 pre-implantation embryos, in post-implantation embryonic and extra-embryonic (placenta) tissues at around day 40 of gestation and in full term offspring. In general, we observe a de-methylation of the DMR at early embryonic stages (day 17) of development followed by a partial or complete re-methylation of the affected DMR of all three imprinted genes at the fetal stages (day 40-50) of development (Figure 1).

\section{REPROGRAMMING OF THE SNRPN GENOMIC IMPRINT}

Methylation patterns of the SNRPN DMR are affected in SCNT, whereas no differences were observed between in vivo- and in vitro-derived day 17 embryos (Lucifero et al., 2006), suggesting that this epigenetic anomaly is caused by an erroneous repro- gramming of the somatic cell chromatin by the oocyte cytoplasm and not by the culture conditions employed during initial development to the blastocyst stage. In follow-up experiments we confirmed that the SNRPN DMR demethylation of SCNT is also observed after implantation, in both embryonic (brain, heart, liver, and skeletal muscle) and extra-embryonic (placenta) tissues (Suzuki et al., 2009). Surprisingly, a less prominent hypomethylation of the SNRPN DMR was also observed in in vitro-derived embryonic and extraembryonic tissues, indicating that in vitro culture during early development can induce epigenetic errors that persist beyond implantation. Indeed, having identified a Bos indicus SNP within exon 2 of SNRPN, we demonstrated that whereas in vivo-derived day-17 are monoallelic, i.e., expressing exclusively from paternal allele, both in vitro and SCNT embryos showed significantly higher levels of maternal expression. However, in vitro-derived bi-allelic expression was no longer observed in embryonic tissues after implantation (day 45), suggesting that the hypomethylation patterns observed in these 
tissues are insufficient to induce maternal expression. Nonetheless, placental tissues remained bi-allelic beyond implantation, indicating that the extraembryonic tissue may sustain higher levels of hypomethylation. In contrast, SCNT-derived embryonic and extra-embryonic tissues showed substantial bi-allelic expression and DMR hypomethylation, indicating that even embryos with severe epigenetic anomalies to SNRPN can survive beyond implantation.

\section{REPROGRAMMING OF THE H19/IGF2 GENOMIC IMPRINT}

To extend our finding to other bovine imprinted loci, we identified SNPs in the DMR and the transcript (cDNA) of the H19 gene in cattle (Suzuki et al., 2011). Analysis of the DMR of the maternally expressed $H 19$ gene indicated a hypermethylation of the paternal allele in the CTCF binding site. Similar to SNRPN, SCNT caused the hypomethylation of the imprinted paternal allele in day 17 pre-implantation embryos, which correlates significantly with increased expression of $\mathrm{H} 19$ from the paternal allele, i.e., bi-allelic expression. Interestingly, compared to the in vivo group, the overall transcript abundance level of both H19 and IGF2 was reduced in SCNT and in vitro-derived embryos. During post-implantation, methylation of the H19 CTCF was not affected in placenta, but was reduced in SCNT embryonic muscle when compared to in vivo and in vitro samples. However, in contrast to the unaffected SNRPN DMR during post-implantation, SCNT led to a partial hypomethylation of the paternal DMR and CTCF binding site on the paternally imprinted allele. Nonetheless, exclusive mono-allelic maternal expression was observed in both embryonic (muscle) and extra-embryonic (placenta) postimplantation tissues, indicating that the hypomethylation levels were insufficient to enable expression from the paternal allele. Interestingly, overall transcript abundance levels of IGF2, but not H19, were slightly increased in the placenta of SCNT-derived gestations. Since SCNT embryos are usually smaller, it is possible that increased IGF2 expression adversely affects the growth rate of the embryo itself.

\section{REPROGRAMMING OF THE IGF2R GENOMIC IMPRINT}

The DMR2 of the bovine IGF2R gene is between 2 to $2.7 \mathrm{~kb}$ in length and is localized in the second intron, approximately $4.4 \mathrm{~kb}$ upstream from exon 2 and $2.2 \mathrm{~kb}$ downstream from exon 3 (Smith et al., 2012). As expected, the DMR is nonmethylated in sperm DNA and hypermethylated in oocytes. With the identification of SNPs in the Bos indicus cattle, we have been able to confirm that the IGF2R DMR in somatic tissues of adult F1 individuals shows hypomethylation of the paternal allele and hypermethylation of the maternal allele. Preliminary analysis of the paternally imprinted IGF2R gene in day 17 embryos indicates that, although the methylation patterns of the DMR are reduced in the SCNT, expression is consistently bi-allelic, regardless of whether the embryos are derived in vivo, in vitro or by SCNT. At day 45 of development, the IGF2R DMR in fetal tissues is consistently hypermethylated whereas the placenta is significantly less methylated, particularly in the SCNT group. Nonetheless, IGF2R biallelic expression is present in all tissues, regardless of the method employed to derive the embryos, i.e., in vivo, in vitro, or SCNT. Therefore, IGF2R seems not to be strongly imprinted at any stage in development in cattle, indicating that an epigenetic mechanism other than the methylation of the DMR may be implicated in controlling allele-specific expression.

\section{EPIGENETIC REPROGRAMMING OF GENOMIC IMPRINTS IN POSTNATAL CLONES}

Abnormal methylation patterns have been observed in cloned ruminants, and these epigenetic anomalies have been associated with developmental effects that lead to postnatal mortality, i.e., the LOS syndrome (Young et al., 2003; Curchoe et al., 2009). To further examine whether LOS derived bovine clones show imprinted gene defects using our allele-specific analysis, we analyzed the paternal and maternal methylation patterns of the H19 and IGF2R DMRs and expression patterns. In contrast to previous reports, adult somatic tissues of cloned calves that died within $24 \mathrm{~h}$ after birth showed normal levels of methylation relative to healthy clones and to animals born by artificial insemination. Moreover, maternal IGF2R DMRs in tissues from in vivo and cloned individuals are consistently hypermethylated, regardless of whether the clones were born in good health. However, levels of IGF2R bi-allelic expression proved to be higher in in vivoderived than clones. Together, these results indicate that SCNTderived animals that develop to term show normal methylation of the H19 imprinted locus, regardless of their health condition at birth.

\section{EPIGENETIC REPROGRAMMING OF GENOMIC IMPRINTS IN INDUCED PLURIPOTENT STEM CELLS}

Apart from SCNT, reversion to pluripotency in somatic cells has been achieved through the induction with pluripotency-related factors, resulting in induced pluripotent stem (iPS) cells. Recent preliminary studies with bovine iPS cell lines developed from F1 hybrid (B. taurus $\times B$. indicus) fetal fibroblasts using transduction with a polycistronic excisable lentivirus containing mouse Oct4, Sox $c$-Myc, and Klf-4 transcription factors have indicated the H19 and SNRPN DMRs are hypomethylated in some iPS lines (Figure 2; Bressan et al., 2014). Moreover, gene expression analyses revealed a bi-allelic expression of $\mathrm{H} 19$ and decreased global expression of both $\mathrm{H} 19$ and IGF2 in most iPS lines. However, $S N R P N$ transcripts were exclusively monoallelic regardless of a significant increase in global expression of SNRPN. Together, these preliminary results indicate that genetically induced reprogramming of somatic cells can lead to epigenetic alterations to bovine imprinted genes in a manner similar to SCNT and in vitro embryo production, supporting the use of iPS cells as a model system to analyze the effects of ART on imprinted genes.

\section{CONCLUSIONS AND FUTURE PERSPECTIVES}

In general, DMRs of SNRPN, H10/IGF2, and IGF2R imprinted genes are extensively hypomethylated in the early stage embryos derived by SCNT and to a lesser extent also in IVP embryos, indicating that the reprogramming of the chromatin and the culture in vitro of oocytes and or embryos causes epigenetic erasure of imprinted loci. Moreover, fewer epigenetic anomalies are observed at later stages of development, suggesting that the imprinted DMRs are either re-methylated during development 


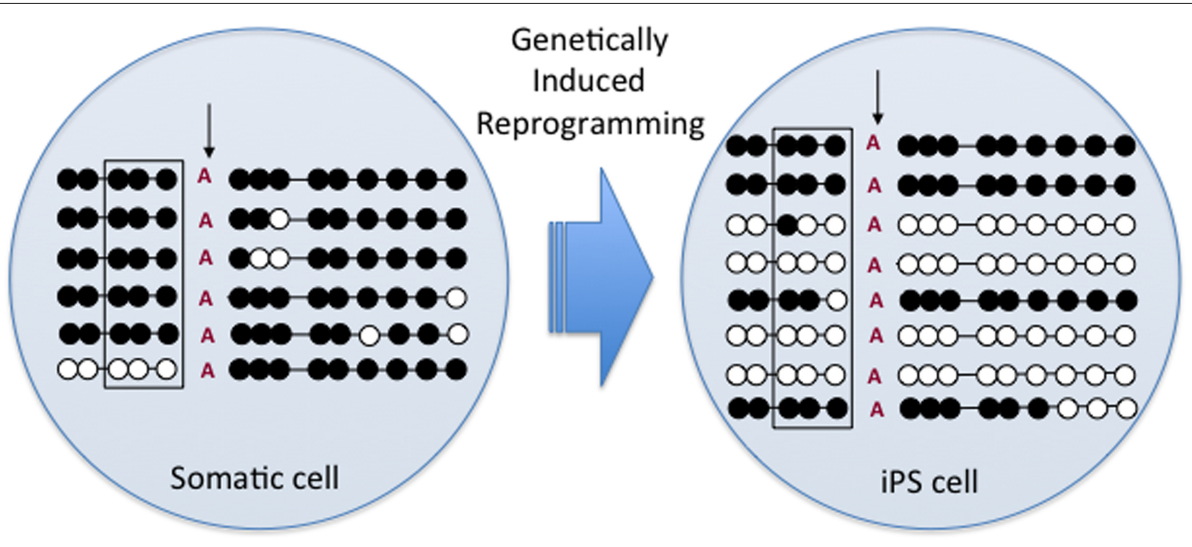

FIGURE 2 | DNA methylation pattern of the paternal H19/IGF2 DMR in hybrid fibroblasts (somatic cell) and induced pluripotent stem (iPS) cells. Circles represent methylated (black) and non-methylated (white) CpGs on the paternal allele DMR as confirmed by a Bos indicus-specific SNP (vertical arrow). Square indicates location of the CTCF binding site also indicating significant de-methylation after iPS cell reprogramming. or that only the less affected embryos survive to later stages of gestation and to term.

Epigenetic errors are likely to have significant effects on the prenatal and postnatal phenotype of domestic animals. Strategies aiming to modify the epigenome by promoting or inhibiting methylation/acetylation drugs prior or during SCNT have been used, however, results remain inconsistent (Sangalli et al., 2014). Since evidence is mounting that show some epigenetic errors result from handling gametes and early embryos in vitro, particularly with cloning by SCNT, further care must be taken when utilizing ART to improve the reproductive performance or accelerate the dissemination of valuable livestock genetic material. However, animals that develop to term seem to retain normal methylation patterns of regulatory regions that control the expression of imprinted genes regardless of their health status, suggesting that embryos carrying harmful epimutations may be selectively eliminated before birth. On the other hand, epigenetic variants that improve survival could be useful for generating animals with increased efficiency. Finally, epigenetic modifications to animals derived by ART could bring transgenerational advantages and risks to animal breeding programs.

\section{REFERENCES}

Bagnicka, E., Siadkowska, E., Strzalkowska, N., Zelazowska, B., Flisikowski, K., Krzyzewski, J., et al. (2010). Association of polymorphisms in exons 2 and 10 of the insulin-like growth factor 2 (IGF2) gene with milk production traits in Polish Holstein-Friesian cattle. J. Dairy Res. 77, 37-42. doi: 10.1017/ S0022029909990197

Bartholdi, D., Krajewska-Walasek, M., Ounap, K., Gaspar, H., Chrzanowska, K. H., Ilyana, H., et al. (2009). Epigenetic mutations of the imprinted IGF2-H19 domain in Silver-Russell syndrome (SRS): results from a large cohort of patients with SRS and SRS-like phenotypes. J. Med. Genet. 46, 192-197. doi: 10.1136/ jmg.2008.061820

Bressan, F. F., Therrien, J., Filion, F., Perecin, F., Smith, L. C., and Meirelles, F. V. (2014). 331 abnormal DNA methylation patterns and allele-specific expression of imprinted genes in bovine-induced pluripotent stem cells. Reprod. Fertil. Dev. 27, 254. doi: 10.1071/RDv27n1Ab331

Brisville, A. C., Fecteau, G., Boysen, S., Dorval, P., Buczinski, S., Blondin, P., et al. (2011). Respiratory disease in neonatal cloned calves. J. Vet. Intern. Med. 25, 373-379. doi: 10.1111/j.1939-1676.2011.0693.x
Brown, K. W., Villar, A. J., Bickmore, W., Clayton-Smith, J., Catchpoole, D., Maher, E. R., et al. (1996). Imprinting mutation in the Beckwith-Wiedemann syndrome leads to biallelic IGF2 expression through an H19-independent pathway. Hum. Mol. Genet. 5, 2027-2032. doi: 10.1093/hmg/5.12.2027

Buczinski, S., Fecteau, G., Lefebvre, R. C., and Smith, L. C. (2011). Assessment of fetal well-being in cattle by ultrasonography in normal, high-risk, and cloned pregnancies. Can. Vet. J. 52, 136-141.

Ceelen, M., and Vermeiden, J. P. (2001). Health of human and livestock conceived by assisted reproduction. Twin Res. 4, 412-416. doi: 10.1375/136905201 2614

Chen, Z., Robbins, K. M., Wells, K. D., and Rivera, R. M. (2013). Large offspring syndrome: a bovine model for the human loss-of-imprinting overgrowth syndrome Beckwith-Wiedemann. Epigenetics 8, 591-601. doi: 10.4161/epi. 24655

Constant, F., Guillomot, M., Heyman, Y., Vignon, X., Laigre, P., Servely, J. L., et al. (2006). Large offspring or large placenta syndrome? Morphometric analysis of late gestation bovine placentomes from somatic nuclear transfer pregnancies complicated by hydrallantois. Biol. Reprod. 75, 122-130. doi: 10.1095/ biolreprod.106.051581

Curchoe, C. L., Zhang, S., Yang, L., Page, R., and Tian, X. C. (2009). Hypomethylation trends in the intergenic region of the imprinted IGF2 and H19 genes in cloned cattle. Anim. Reprod. Sci. 116, 213-225. doi: 10.1016/j.anireprosci. 2009.02.008

Daxinger, L., and Whitelaw, E. (2012). Understanding transgenerational epigenetic inheritance via the gametes in mammals. Nat. Rev. Genet. 13, 153-162. doi: $10.1038 / \mathrm{nrg} 3188$

Flisikowski, K., Adamowicz, T., Strabel, T., Jankowski, T., Switonski, M., and Zwierzchowski, L. (2007). An InDel polymorphism in exon 6 of IGF2 associated with the breeding value of Polish Holstein-Friesian bulls. Biochem. Genet. 45, 139-143. doi: 10.1007/s10528-006-9071-9

Gärtner, K., Bondioli, K. R., Hill, K., and Rapp, K. (1998). High variability of body sizes within nucleus-transfer-clones of calves: artifacts or a biological feature? Reprod. Domest. Anim. 33, 67-75. doi: 10.1111/j.1439-0531.1998.tb 01317.x

Goddard, M. E., and Whitelaw, E. (2014). The use of epigenetic phenomena for the improvement of sheep and cattle. Front. Genet. 5:247. doi: 10.3389/fgene. 2014.00247

Goodall, J. J., and Schmutz, S. M. (2007). IGF2 gene characterization and association with rib eye area in beef cattle. Anim. Genet. 38, 154-161. doi: 10.1111/ j.1365-2052.2007.01576.x

Grandjean, V., Gounon, P., Wagner, N., Martin, L., Wagner, K. D., Bernex, F., et al. (2009). The miR-124-Sox9 paramutation: RNA-mediated epigenetic control of embryonic and adult growth. Development 136, 3647-3655. doi: 10.1242/dev. 041061

Heard, E., and Martienssen, R. A. (2014). Transgenerational epigenetic inheritance: myths and mechanisms. Cell 157, 95-109. doi: 10.1016/j.cell.2014.02.045 
Heyman, Y., Chavatte-Palmer, P., LeBourhis, D., Camous, S., Vignon, X., and Renard, J. P. (2002). Frequency and occurrence of late-gestation losses from cattle cloned embryos. Biol. Reprod. 66, 6-13. doi: 10.1095/biolreprod66.1.6

Heyman, Y., Richard, C., Rodriguez-Martinez, H., Lazzari, G., Chavatte-Palmer, P., Vignon, X., et al. (2004). Zootechnical performance of cloned cattle and offspring: preliminary results. Cloning Stem Cells 6, 111-120. doi: 10.1089/ 1536230041372364

Hill, J. R. (2002). Abnormal in utero development of cloned animals: implications for human cloning. Differentiation 69, 174-178. doi: 10.1046/j.1432-0436.2002. 690408.x

Kohan-Ghadr, H. R., Fecteau, G., Smith, L. C., Murphy, B. D., and Lefebvre, R. C. (2011). Endocrine profiles of somatic nuclear transfer-derived pregnancies in dairy cattle. Theriogenology 76, 911-920. doi: 10.1016/j.theriogenology. 2011.04.022

Lucifero, D., Suzuki, J., Bordignon, V., Martel, J., Vigneault, C., Therrien, J., et al. (2006). Bovine SNRPN methylation imprint in oocytes and day 17 in vitroproduced and somatic cell nuclear transfer embryos. Biol. Reprod. 75, 531-538. doi: 10.1095/biolreprod.106.051722

Lumey, L. H., Stein, A. D., Kahn, H. S., and Romijn, J. A. (2009). Lipid profiles in middle-aged men and women after famine exposure during gestation: the Dutch Hunger Winter Families Study. Am. J. Clin. Nutr. 89, 1737-1743. doi: 10.3945/ajcn.2008.27038

Magee, D. A., Spillane, C., Berkowicz, E. W., Sikora, K. M., and MacHugh, D. E. (2014). Imprinted loci in domestic livestock species as epigenomic targets for artificial selection of complex traits. Anim. Genet. 45(Suppl. 1), 25-39. doi: 10.1111/age. 12168

Maher, E. R., Afnan, M., and Barratt, C. L. (2003). Epigenetic risks related to assisted reproductive technologies: epigenetics, imprinting, ART and icebergs? Hum. Reprod. 18, 2508-2511. doi: 10.1093/humrep/deg486

Meirelles, F. V., Birgel, E. H., Perecin, F., Bertolini, M., Traldi, A. S., Pimentel, J. R., et al. (2010). Delivery of cloned offspring: experience in Zebu cattle (Bos indicus). Reprod. Fertil. Dev. 22, 88-97. doi: 10.1071/RD09229

Morgan, H. D., Sutherland, H. G., Martin, D. I., and Whitelaw, E. (1999). Epigenetic inheritance at the agouti locus in the mouse. Nat. Genet. 23, 314-318. doi: $10.1038 / 15490$

Ng, S. F., Lin, R. C., Laybutt, D. R., Barres, R., Owens, J. A., and Morris, M. J. (2010). Chronic high-fat diet in fathers programs beta-cell dysfunction in female rat offspring. Nature 467, 963-966. doi: 10.1038/nature09491

Painter, R. C., Osmond, C., Gluckman, P., Hanson, M., Phillips, D. I., and Roseboom, T. J. (2008). Transgenerational effects of prenatal exposure to the Dutch famine on neonatal adiposity and health in later life. BJOG 115, 1243-1249. doi: 10.1111/j.1471-0528.2008.01822.x

Pembrey, M. E., Bygren, L. O., Kaati, G., Edvinsson, S., Northstone, K., Sjostrom, M., et al. (2006). Sex-specific, male-line transgenerational responses in humans. Eur. J. Hum. Genet. 14, 159-166. doi: 10.1038/sj.ejhg.5201538

Reed, M. L., and Leff, S. E. (1994). Maternal imprinting of human SNRPN, a gene deleted in Prader-Willi syndrome. Nat. Genet. 6, 163-167. doi: 10.1038/ng0294163

Runte, M., Kroisel, P. M., Gillessen-Kaesbach, G., Varon, R., Horn, D., Cohen, M. Y., et al. (2004). SNURF-SNRPN and UBE3A transcript levels in patients with Angelman syndrome. Hum. Genet. 114, 553-561. doi: 10.1007/s00439-004$1104-\mathrm{z}$

Sangalli, J. R., Chiaratti, M. R., De Bem, T. H., de Araujo, R. R., Bressan, F. F., Sampaio, R. V., et al. (2014). Development to term of cloned cattle derived from donor cells treated with valproic acid. PLoS ONE 9:e101022. doi: 10.1371/journal.pone. 0101022

Sherman, E. L., Nkrumah, J. D., Murdoch, B. M., Li, C., Wang, Z., Fu, A., et al. (2008). Polymorphisms and haplotypes in the bovine neuropeptide Y, growth hormone receptor, ghrelin, insulin-like growth factor 2, and uncoupling proteins 2 and 3 genes and their associations with measures of growth, performance, feed efficiency, and carcass merit in beef cattle. J. Anim. Sci. 86, 1-16. doi: 10.2527/jas.2006-799
Smith, L. C., Suzuki, J. Jr., Goff, A. K., Filion, F., Therrien, J., Murphy, B. D., et al. (2012). Developmental and epigenetic anomalies in cloned cattle. Reprod. Domest. Anim. 47(Suppl. 4), 107-114. doi: 10.1111/j.1439-0531.2012.02063.x

Stinckens, A., Mathur, P., Janssens, S., Bruggeman, V., Onagbesan, O. M., Schroyen, M., et al. (2010). Indirect effect of IGF2 intron3 g.3072G $>$ A mutation on prolificacy in sows. Anim. Genet. 41, 493-498. doi: 10.1111/j.1365-2052.2010. 02040.x

Suzuki, J. Jr., Therrien, J., Filion, F., Lefebvre, R., Goff, A. K., Perecin, F., et al. (2011). Loss of methylation at H19 DMD is associated with biallelic expression and reduced development in cattle derived by somatic cell nuclear transfer. Biol. Reprod. 84, 947-956. doi: 10.1095/biolreprod.110.087833

Suzuki, J. Jr., Therrien, J., Filion, F., Lefebvre, R., Goff, A. K., and Smith, L. C. (2009). In vitro culture and somatic cell nuclear transfer affect imprinting of SNRPN gene in pre- and post-implantation stages of development in cattle. BMC Dev. Biol. 9:9. doi: 10.1186/1471-213X-9-9

Urrego, R., Rodriguez-Osorio, N., and Niemann, H. (2014). Epigenetic disorders and altered gene expression after use of Assisted Reproductive Technologies in domestic cattle. Epigenetics 9, 803-815. doi: 10.4161/epi.28711

van der Heijden, G. W., Ramos, L., Baart, E. B., van den Berg, I. M., Derijck, A. A., van der Vlag, J., et al. (2008). Sperm-derived histones contribute to zygotic chromatin in humans. BMC Dev. Biol. 8:34. doi: 10.1186/1471-213X-8-34

Van Laere, A. S., Nguyen, M., Braunschweig, M., Nezer, C., Collette, C., Moreau, L., et al. (2003). A regulatory mutation in IGF2 causes a major QTL effect on muscle growth in the pig. Nature 425, 832-836. doi: 10.1038/nature 02064

Vykoukalova, Z., Knoll, A., Dvorak, J., and Cepica, S. (2006). New SNPs in the IGF2 gene and association between this gene and backfat thickness and lean meat content in Large White pigs. J. Anim. Breed Genet. 123, 204-207. doi: 10.1111/ j.1439-0388.2006.00580.x

Wells, D. N., Forsyth, J. T., McMillan, V., and Oback, B. (2004). The health of somatic cell cloned cattle and their offspring. Cloning Stem Cells 6, 101-110. doi: 10.1089/1536230041372300

Wykes, S. M., and Krawetz, S. A. (2003). The structural organization of sperm chromatin. J. Biol. Chem. 278, 29471-29477. doi: 10.1074/jbc.M304545200

Young, L. E., Fernandes, K., McEvoy, T. G., Butterwith, S. C., Gutierrez, C. G., Carolan, C., et al. (2001). Epigenetic change in IGF2R is associated with fetal overgrowth after sheep embryo culture. Nat. Genet. 27, 153-154. doi: 10.1038/ 84769

Young, L. E., Schnieke, A. E., McCreath, K. J., Wieckowski, S., Konfortova, G., Fernandes, K., et al. (2003). Conservation of IGF2-H19 and IGF2R imprinting in sheep: effects of somatic cell nuclear transfer. Mech. Dev. 120, 1433-1442. doi: 10.1016/j.mod.2003.09.006

Young, L. E., Sinclair, K. D., and Wilmut, I. (1998). Large offspring syndrome in cattle and sheep. Rev. Reprod. 3, 155-163. doi: 10.1530/ror.0.0030155

Conflict of Interest Statement: The authors declare that the research was conducted in the absence of any commercial or financial relationships that could be construed as a potential conflict of interest.

Received: 18 September 2014; accepted: 07 February 2015; published online: 25 February 2015.

Citation: Smith LC, Therrien J, Filion F, Bressan F and Meirelles FV (2015) Epigenetic consequences of artificial reproductive technologies to the bovine imprinted genes SNRPN, H19/IGF2, and IGF2R. Front. Genet. 6:58. doi: 10.3389/fgene.2015.00058

This article was submitted to Livestock Genomics, a section of the journal Frontiers in Genetics.

Copyright (C) 2015 Smith, Therrien, Filion, Bressan and Meirelles. This is an openaccess article distributed under the terms of the Creative Commons Attribution License (CC BY). The use, distribution or reproduction in other forums is permitted, provided the original author(s) or licensor are credited and that the original publication in this journal is cited, in accordance with accepted academic practice. No use, distribution or reproduction is permitted which does not comply with these terms. 